\title{
Remote Patient Health Monitoring System Using Mobile and Wireless Body Area Network in Yemen
}

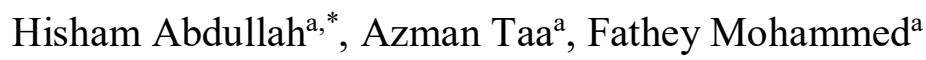 \\ ${ }^{a}$ School of Computing, Universiti Utara Malaysia (UUM), 06010 Sintok, Kedah Darul Aman, Malaysia \\ Corresponding author: ${ }^{*}$ h.alruaini@gmail.com
}

\begin{abstract}
The traditional approach for monitoring chronic diseases represents a major challenge for hospitals and the Ministry of Health in Yemen. This research aims to develop a Remote Patient Health Monitoring System (RPHMS) using Bluetooth enabled medical sensors and mobile phones. This research adopts the Rational Unified Process (RUP) is a software development process framework to develop the proposed system. The RPHMS provides real-time monitoring for the patients suffering from chronic diseases and manages the patients' medical record in which the diagnostic and prescriptive data are stored for further analysis. The RPHMS consists of two major subsystems: Mobile Patient System (MPS) to wirelessly measure the patient's medical and position data, and automatically send the data to the central hospital server; and Central Hospital System (CHS) to automatically store the diagnostic data in the hospital database and then forward the data in form of Short Message Service (SMS) to the doctor. In addition, the CHS enables the patient to get feedback from the doctor. Moreover, CHS stores the feedback and the prescriptions in the hospital database. These data can be accessed by the patient using a mobile application. The results show that RPHMS enables hospitals and doctors to obtain accurate and timely data regarding patients' chronic illnesses. This research concludes that the RPHMS improves the control of the heart rate, blood pressure, and oxygen percentage in the blood (SPO2), since it provides real-time monitoring of chronic patients.
\end{abstract}

Keywords - Remote Patient Monitoring System (RPMS); Wireless Body Area Network (WBAN); mobile technology.

Manuscript received 12 Oct. 2020; revised 5 Dec. 2020; accepted 10 Dec. 2020. Date of publication 28 Feb. 2021. IJASEIT is licensed under a Creative Commons Attribution-Share Alike 4.0 International License.

\section{INTRODUCTION}

The Wireless Body Area Network (WBAN) is a sensing technology that can be continuously worn on the patient's body. The primary purpose of WBAN is to monitor the health situation of patients. It consists of several types of medical sensors. For example, wearable medical sensors are used to form the WBAN around the body of the patient. The medical sensors of WBAN are characterized by low power, small size, and diffusive wireless sensors [1], [2]. The fast development of the WBAN has played a big role in providing efficient healthcare facilities to the sufferers of chronic illnesses. Besides, mobile technology provides the fastest way to deliver the patient's data to the doctor. Therefore, the development of healthcare applications based on WBAN together with mobile devices may assist millions of people who suffer from chronic illnesses by improving the quality of their lives and reducing healthcare costs [2], [3]. Additionally, the increase of chronic diseases poses an urgent need to develop mobile healthcare applications to monitor the patients in remote areas [4]. The present knowledge of mobile healthcare applications is still limited. More specifically, most of the previous studies that have been conducted on mobile health monitoring systems focused on the following two solutions:

- Measuring and transmitting of patient's data to the hospital server or the doctor.

- Sending the feedback from the doctor's mobile phone to the patient's mobile phone directly.

However, these solutions still cannot provide a sufficient interest for the patients from two aspects: firstly, the data of patients lack security and privacy, particularly in case of missing doctor's mobile phone. Besides, the patient could not ensure if that message came from the doctor or not. Secondly, the medical prescriptions are not permanently stored in the patient's medical record for future diagnosis and decision-making. On the other hand, those studies ignored the process of storing patients' prescriptions in the database of the remote hospital server. The data of patients can be sent via wireless communication such as the General Pocket 
Radio Service (GPRS) technology (2.5G), which is provided by telecommunication companies [5], [6].

SMS is considered a better communication way of mobile technology than web service and socket technologies. The SMS communication protocol does not resemble web services that need to be hosted by the service provider for applying a request and response communication model. SMS messages can be sent and received as and when needed. Some studies indicated the importance of collecting the patient's location data by using the Global Positioning System (GPS) receiver. The GPS receiver is considered a feasible technology to identify the current location of the patient. It provides the hospital with the location data of the patient. These data enable the nurse to visit the patient's current place and enable the healthcare provider to bring the patient to the hospital. This is necessary when the remote medical instructions are not useful. Before the actual monitoring process, the patient must subscribe to the hospital or healthcare center to obtain the remote monitoring service [7]. Many prior studies lack developing the Patient Subscription System (PSS) as an integral subsystem of the central hospital system. This does not mean that the PSS is not an issue in remote health monitoring applications. Nevertheless, the PSS is an essential system to be implemented in the central hospital server since it improves the hospital's service quality. It also enables the healthcare provider to manage and process the data of patients' subscriptions.

The rapid spreading and increasing of chronic diseases represent a significant challenge to the Ministry of Health and Yemen hospitals. For instance, the University and Science Technology Hospital (USTH) stated that more than $40 \%$ of primary visits to the hospital related to chronic illnesses [8]. Furthermore, World Health Organization (WHO) found that there are about 330 million individuals have hypertension. Accordingly, in 2025, it might be around 1.56 billion individuals suffering from high blood pressure [9]. However, all current monitoring systems implemented in the private and public hospitals in Yemen are still using the traditional approach of monitoring chronic patients. For instance, when the medical professionals or the doctors want to monitor the patients, they visit the patients at their beds to make a suitable medical diagnosis. However, it is hard for the patients to stay throughout their life in the hospital, and it is also difficult for the doctor to visit the patient [1] continuously.

Regarding chronic patients, there is a necessity to maintain their health situation under monitoring and control. Besides, many hospitals do not have an adequate capacity to accommodate all chronic patients. Accordingly, the manual approach is not suitable to monitor and manage these patients.

Besides, storing the medical diagnosis data and patients permanently in the remote hospital server database is vital to future diagnosis and decision-making. However, the patient centrally, the manual approach fails to address this issue [1]. Furthermore, many prior studies developed the patient's medical record, which did not provide an adequate solution for storing and managing the following: medical prescriptions and diagnosis of patients, time of receiving of patient's diagnosis, and time of sending of feedback to the patient [5]. An effective way of managing chronic diseases can provide improved healthcare results. For example, monitoring the patient's heart rate, blood pressure, and SPO2 can improve quality of life and reduce health costs [10].

According to Evans et al. [11], the healthcare applications of the WBAN are divided into two categories relying on the environments of their operating:

- Wearable WBAN (i.e., wearable sensors) work on the patients' body such as portable, ambulatory, and embedded sensors. They are used for the medical sensing and monitoring.

- Implantable WBAN (i.e., implantable sensors), which work inside the patient's body.

Accordingly, this research utilizes portable medical sensors known as ECG [1], [12], [13]. This medical sensor is incorporated with Bluetooth technology as a communication protocol. It has enough bit rate (up to $433.9 \mathrm{~Kb} / \mathrm{s}$ ) and bandwidth to transmit the mobile phones' signals. It also enables the data to be transmitted securely because it supports authentication and encryption methods. The previous paragraphs discuss that WBAN is a sufficient sensing technology for healthcare monitoring systems [14], [15].

The remote healthcare systems have been developed and implemented in Africa, Europe, and America countries. Some of them were developed to support healthcare monitoring for remote patients at home. Many types of research are being done to review and improve the current systems [4]. Many remote patients monitoring projects were developed and implemented to be used for monitoring patients at home, particularly those who have lost their mobility [16], [17]. They suggest that the patients' data can be delivered to the healthcare center if the patient resides at home. However, anywhere delivery of the patient's data is not supported by those systems. Another similar previous study focuses on sensing and sending patients' data directly from PDA to the doctors [18], [19]. They propose the system, which is named "Remote Patient Monitoring" (RPM). It is integrating GPS, GSM and wireless sensors have been developed. It uses the plus oximeter sensor and cellular phone to send patients' data in SMS to the doctors' mobile phones. The SMS message contains the patient's medical and location information. The RPM focuses on sensing and sending the patient's data directly to the doctors. However, the RPM does not have patients' medical modules located in hospitals. It also does not have security protection over the patients' data.

Regarding the process of real-time monitoring, the health monitoring system called "Enhanced Personal, Intelligent and Mobile System for Early Detection and Interpretation of Cardiological Syndromes" (EPI-MEDICS) is developed to support the real-time monitoring [20]. This system monitors the remote patients at home and their work offices and on trips. They argue about the efficiency of the current remote health monitoring systems and they consider them inadequate to meet the patients' requirements [21]. EPIMEDICS is used to collect the patients' health data and location information and then send the patient's data to alarm the server via mobile phone. The alarm server receives the patients' data together with the alarm message. 
Furthermore, the alarm server forwards the data in the form of SMS to the hospital or to the doctor. The alarm message is understood according to the alarm level. It is necessary to notify the healthcare practitioners (doctors) of the critical signal in a critical situation [13]. However, locating the alarm software in a separate server (such as an alarm server) is inefficient and slows down performance [22]. Indeed, the data need more management and processing until it received by the doctors. It is better to embed the alarm software as an integral subsystem of the hospital software. Another issue is that the patients' feedback is not stored in the patient's medical record. It is a big limitation. Commonly, the software in the hospitals consists of two subsystems:

- The patient's medical record, which processes and manages the information and history of the patients.

- GPS module that processes and manages locations information of the patients and enables doctors to explore patients' current location.

On the other hand, the SMS technology is not used as a communication protocol in some previous projects [23]. They justified that the SMS needs interaction from the patient to retrieve messages [23]. However, there are studies focusing on the importance of using SMS and MMS as a communication medium in the healthcare monitoring application [7]. For example, some previous research argues that mobile SMS and the MMS in healthcare monitoring systems are essential for the patients until they can benefit less time, effort, and cost [24].

At present, the hospitals and healthcare centers in Yemen need to monitor their chronic patients systematically. Therefore, new solutions are required. This study aims to develop the RPHMS that can play an essential role in providing healthcare services for remote patients, particularly those in the Yemeni rural areas.

\section{MATERIALS AND METHOD}

This section discusses the proposed system model, the tools, the processes, the techniques, and methods used to design, develop, and implement the system. The RUP of the system development life cycle is the methodology that is used in the analysis, design, and implementation of the RPHMS. The proposed model of RPHMS is shown in Fig. 1.

\section{A. The Proposed Model}

As shown in Fig. 1, the RPHMS mainly consists of two systems: the MPS and the CHS. Firstly, the MPS is a mobile system installed in the patient's mobile phone to measure the patient's data from wearable sensor devices and then send the data to the hospital medical server. Secondly, the CHS is the system that will be installed in the remote central server. It is used for receiving the patients' data and forwarding the data to the doctor. Furthermore, the CHS consists of four subsystems: the PMR, the PSS, the SMS system, and the PTS. Firstly, the MPS is a mobile application that is proposed to be installed on the patient's mobile phone. It is responsible for interfacing between the wearable body sensors and the hospital server. It also measures the patient's data from the wearable sensors and sends the patient's data to the hospital server. Secondly, the CHS software consists of four subsystems as follow: PSS, PMR, SMS management system, and PTS.
1) The PSS: it is proposed to serve the subscription phase such as patients' subscription and personal data, subscription duration, subscription fees and the remote monitoring services. The PSS also manages and controls the configurations of the patients' medical sensors. Besides, it authenticates the patients' access to accept or reject the patients' data. Furthermore, it stores, manages, and controls the users' accounts and performs user validation.

2) The PMR: it is proposed to manage the patient's medical record. It includes the patient's diagnostic data received from the patient's medical sensors and the doctor's prescriptions for the patient. The patient's diagnostic data and the patient's prescription information must be automatically inserted and saved into the patient's medical record.

3) The SMS: SMS management system is proposed to act as a communication ring between the patient and the doctor. It is used to receive the patient's data from the patient's mobile phone and forward the medical data to the doctor responsible for monitoring the patient. It also used to receive feedback from the doctor and forward the feedback to the patient. Furthermore, the SMS messaging system enables the patient to query about his/her medical record by sending the query results to the patient mobile phone.

4) The PTS: PTS subsystem is proposed to track the patient's location. The PTS stores and manages the patient's position information that is received from the patient's mobile phone. It enables the hospital to identify the current location of the patients. Google's Latitude software will be used to determine the patients' location because it does not need to interact with the vendor or third party.

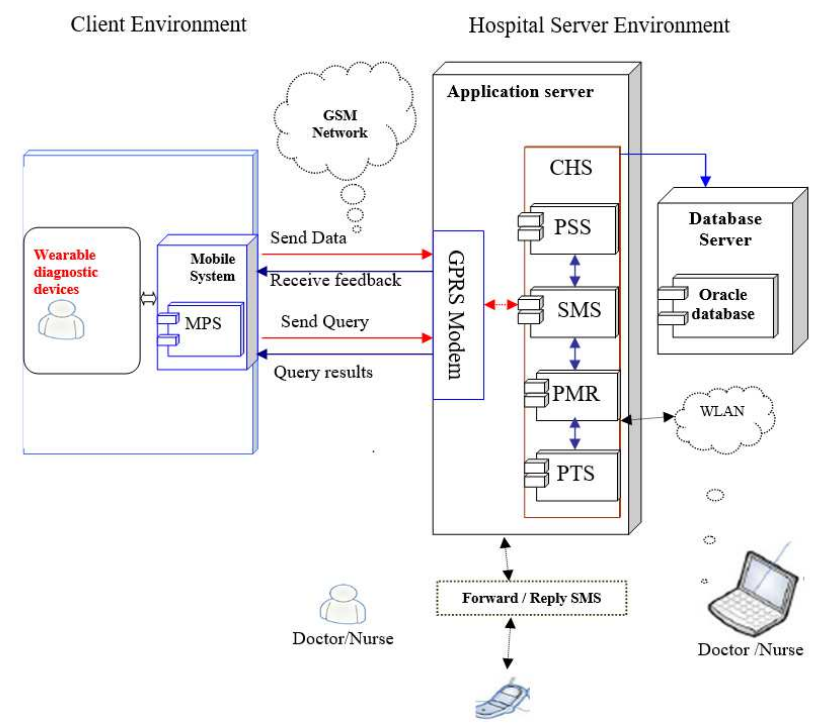

Fig. 1 The proposed model for RPHMS

\section{B. Tools Used for Application Design}

For the server applications, an oracle database $12 \mathrm{C}$ is identified during this phase as the most suitable database for the RPHMS system. An oracle $12 \mathrm{C}$ is selected as an appropriate relational database management system for the proposed system because the researcher has more than 16 years of experience in an oracle database. The reason for 
using oracle database $12 \mathrm{C}$ is that it supports an effective concurrency control model, which involves indexing, partitioning, and parallel execution of processes. Besides, an oracle database is a platform database, which can be run on more than one operating system such as Linux, UNIX, and Windows, and it also supports cloud computing technology [25]. Similarly, Microsoft Visual Studio 2012 (C\#.NET) is used in this phase as an appropriate programming language to develop the Graphical User Interface (GUI) of the RPHMS. It was selected because the researcher has more than 5 years of experience developing GUI by using C\#.NET 2012. As for mobile applications, Windows Mobile, together with C\#.NET 2012, is used for developing mobile applications.

\section{RESULT AND DISCUSSION}

This research determines whether the RPHMS improves the HRV, SPO2, and BP control among patients with uncontrolled diseases in Yemen. The RPHMS has been developed by using mobile phone and GPRS technology. Therefore, it provides the solution of mobility, which enables the patients to be monitored from anywhere and anytime. In addition to the solution of mobility, this study provides other important benefits to the patients, the doctors, and healthcare centers. Furthermore, it met the users' requirements and avoided using the internet technology because a significant obstacle that faces both patients and doctors in Yemen is the unavailability of the internet. The major results of this study's project as follows: the MPS, the SMS, the PMR, the PTS, the PSS, and the results of access into the system.

Firstly, the MPS provides real-time patient's health status and location information. Accordingly, the doctor can monitor the patient's current health status not only at home but also from anywhere and at any time. This result confirms the results of the study developed by previous study [5]. The MPS also displays patient's medical prescriptions messages in a separated list. This may be beneficial to patients so that they can differentiate between medical and other messages.

Secondly, the major results that arose from the implementation of the SMS subsystem are considered very important in improving healthcare services provided by hospitals in terms of the speed of information delivery at the right time and right place. This system works as a liaison between patients and doctors, since it receives the patient's data, stores these data in the database, and sends the patient's medical data to the doctor in charge. After that, it receives a patient's medical prescription transmitted by the doctor, stores it in the database, and sends it to the patient. This result's possible explanation is that the hospital server must authenticate the patient's diagnostic data and the patient's feedback.

Thirdly, the most significant result that emerged from the PMR implementation is that the patients' diagnosis and prescription information are permanently saved for the purposes of the future diagnosis. This means the doctor's feedback must be stored in the patients' medical record before sending the patient's feedback. The availability of the patient's medical record and the patient's history in the PMR could assist the doctors for better treatment.
Fourthly, identifying and browsing the current patient's location is also the significant result of the PTS.

Fifthly, for the hospitals or the healthcare centers, the most significant results which emerge from the PSS are that the healthcare center can manage the patients' subscription procedures such as subscription fees, subscription duration, and monitoring services. Although the patient should be subscribed to the hospital or healthcare center to obtain the remote monitoring service, this enables the patient to ensure the quality of the hospital's remote monitoring service. Therefore, this result provides beneficial value to the hospitals in return to provide a remote monitoring service. In contrast to prior studies, this result has not been previously developed in other studies.

Sixthly, regarding remote access, only the patients and doctors were authorized to access the CHS through using their mobile phones remotely. Their ID and mobile phone number authenticated the doctors while the patients were authenticated by their ID, sensor ID, and mobile phone number. The study developed to confirm that this result is significant due to the patient's medical data's sensitivity [1]. Besides, patients' information must be private and confidential. Regarding local access, the doctor can also access the CHS by using a valid user name and password. The local access into CHS is very useful when the doctor in the hospital. It also enables the doctor to send feedback to the patient using CHS and without using the mobile phone.

\section{A. The Results of The Patient's Subscription}

After registering a new patient by the nurse or doctor in the system, the doctor decides about the chronic diseases of the patient who will be monitored and the required monitoring duration. To assign the remote monitoring services to the patient, the doctor uses the interface given in Fig. 2.

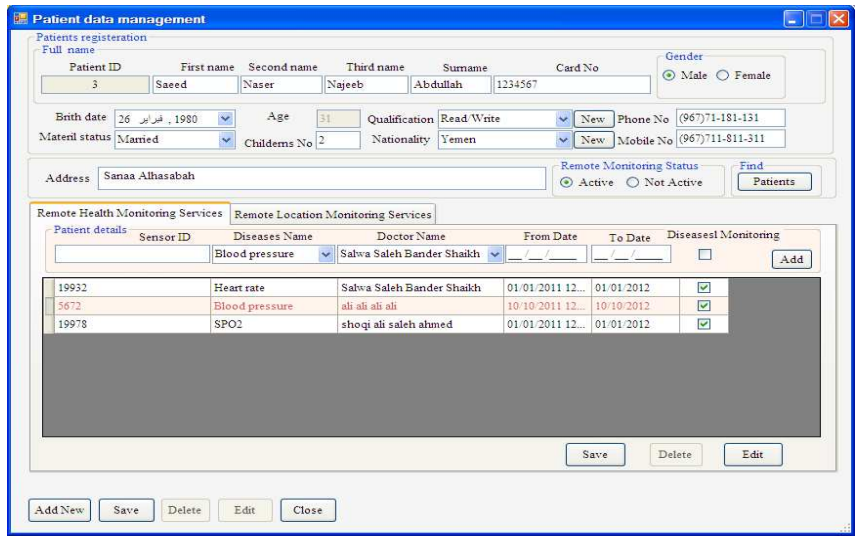

Fig. 2 Assigning the remote monitoring services to the patient (chs interface)

The mandatory fields require to enter as in Figure 2 above are as follows:

- the sensor ID that refers to patient's sensor device identifier.

- The remote monitoring service, which refers to the chronic disease of the patient that will be monitored.

- The user must select the doctor who is responsible for monitoring the patient remotely.

- The beginning date of the patient's subscription, which refers to the start of the monitoring operation. 
- The finishing date of the patient's subscription that refers to the end of the monitoring operation; (6) Enabling of the remote monitoring option, which indicates to confirm the activation of the remote monitoring operation.

For every patient registered in the CHS, the patient's data cannot be accepted by the remote hospital server if the monitoring services are not assigned to the patient and if the patient's monitoring service is not activated.

\section{B. Configuring the MPS for Connecting with the CHS}

After subscribing to the patient in the hospital or healthcare center, the system administrator must install the MPS on the patient's mobile phone. Likewise, the system administrator must prepare the communications settings between the patient's mobile phone and the remote hospital server.

\section{Results of Monitoring Patients Remotely}

This section presents the results of the remote monitoring phase. It starts after registering the communication settings between the client application (MPS) and server application (CHS), and activating the remote monitoring service. In the MPS, the monitoring results are automatically collected from both the medical sensor device and the GPS receiver in one SMS message. In the MPS, the process of measuring realtime health and position information is considered as the significant result of this study. Fig. 3 shows the result of measuring the patient's location information. This figure displays that there are two significant parameters: latitude and longitude.

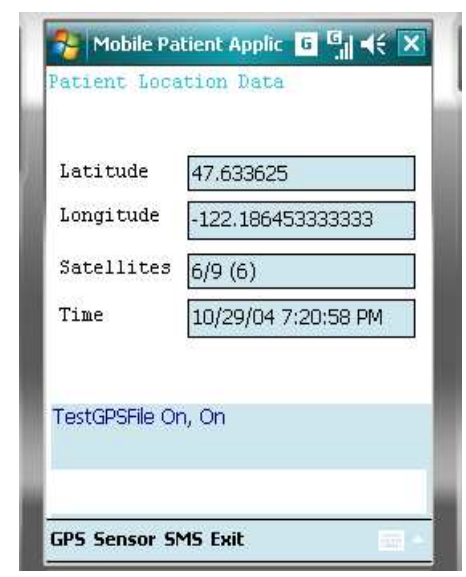

Fig. 3 Patient's Location Data

These parameters are used in identifying the current location of the patient. Fig. 4 displays the result of measuring the patient's medical information. This figure shows that there are three significant parameters: HRV, SPO2, and BP that are used in diagnosing the health status of the patient. In the MPS, the most critical results appear from Fig. 3 and Fig. 4 because the patient's location and medical information are automatically measured without user interaction. Moreover, when the wireless medical sensor's critical values, it directly alerts the MPS to send SMS message to the remote hospital server. The MPS formats the SMS message before transmitting it to the remote hospital server. For the MPS, the SMS message format is implemented to transmit and receive data between the patient's mobile phone and the remote hospital server. This agreement is critical to distinguish the data from a different format as soon as receiving them. Consequently, the data with the different formats will be rejected before the authentication and validation.

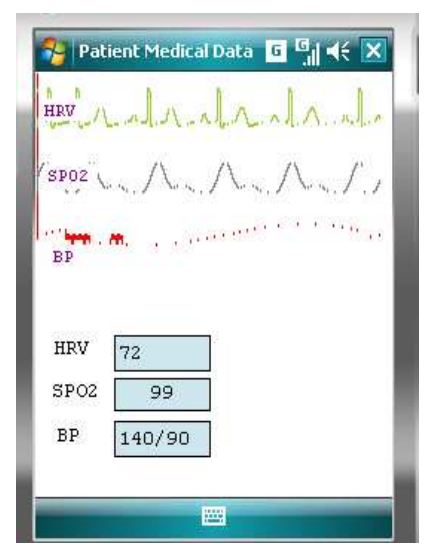

Fig. 4 Patient's medical data

Fig. 5 shows the format of SMS message that sent by the MPS to the remote hospital server. The SMS message begins with the RPHMS identifier followed by the patient ID, sensor ID, medical information, and location information.

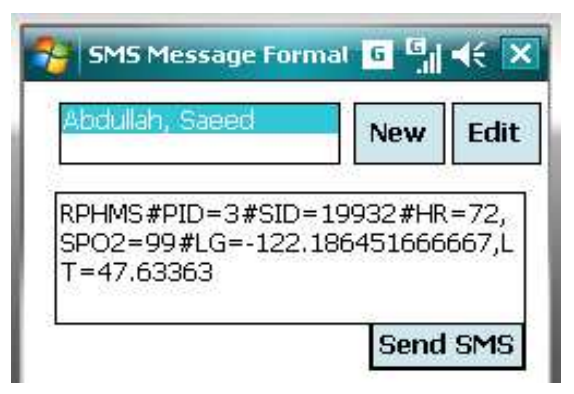

Fig. 5 Transmitting the patient's data in the form of SMS (MPS interface)

While the SMS management system is implemented as a subsystem of the CHS in the remote hospital server, it should be on connectivity and running to serve remote patients. It also works as SMS server to send and receive patient's data and instructions. Fig. 5 shows the interface of the SMS server that consists of four text boxes. These text boxes describe the SMS message status from the top left as shown in Table 1 below.

TABLE I

SMS Message StATUS IN THE CHS

\begin{tabular}{clll}
\hline $\begin{array}{c}\text { Message } \\
\text { Status }\end{array}$ & $\begin{array}{c}\text { Status } \\
\text { description }\end{array}$ & \multicolumn{1}{c}{ From } & To \\
\hline 1 & Received & Patient & $\begin{array}{l}\text { Remote } \\
\text { hospital server } \\
\text { Doctor }\end{array}$ \\
2 & Transmitted & $\begin{array}{l}\text { Remote } \\
\text { hospital server } \\
\text { Doctor }\end{array}$ & $\begin{array}{l}\text { Remote } \\
\text { hospital server } \\
\text { Patient }\end{array}$ \\
\hline
\end{tabular}

Table 1 explains that the SMS message has four statuses in the CHS as follows:

- SMS message that is received from the patient. 
- SMS message that is transmitted to the doctor.

- SMS message that is received from the doctor.

- SMS message that is transmitted to the patient.

In detail, every transmitted SMS message from the patient's mobile phone to the remote hospital server must pass by several steps. The CHS is responsible for performing these steps that are described as follows:

- The CHS examines whether the duration of the patient's participation is not expired. If expired, the CHS will notify the patient of renewing the subscription via SMS message.

- The CHS receives the patient's data in the form of SMS and then displays the received SMS as shown in the interface given in Fig. 6.

- The CHS formats the SMS message by dividing this message into patient's medical data and patient's position data.

- The CHS stores the patient's medical data in the database and displays the results in the patient medical record interface.

- The CHS stores the patient's location data in the database and displays the results in the interface of mapping the patient's location.

- After receiving and storing the patient's data in the database, the patient's medical data is automatically forwarded by the remote hospital server to the related doctor as shown in the interface given in Fig. 6.

- The CHS receives the prescription and instructions for the patient, which was sent from the doctor and stores it in the database.

- The CHS sends the feedback, which was received from the doctor to the related patient and displays the results as shown in the interface given in Fig. 6.

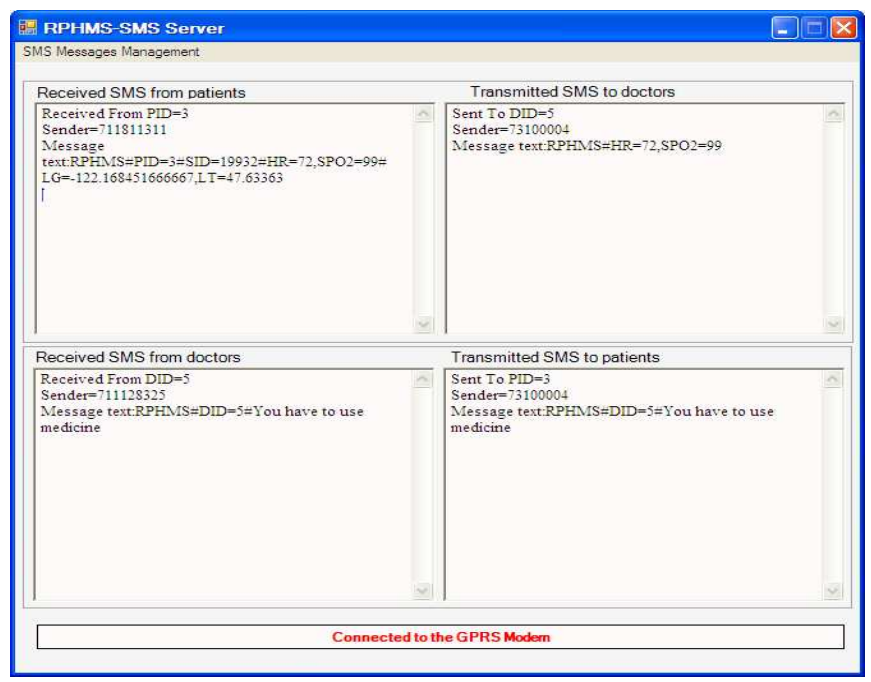

Fig. 6 SMS server for sending/receiving SMS (CHS Interface)

In the CHS, the process of sending and receiving the data may seem long, admittedly, but it benefits the patient greatly. For example, it ensures the storage of the patient's diagnostic data, the medical prescription, the medical instructions, and the medical advice in the patient's medical records. In the MPS, the process of filtering of SMS messages is used to enable the patient to distinguish between the messages, which came from the hospital server and the other SMS messages, which came from others sources. Filtering the
SMS messages that are received by the patient's mobile phone is explained in the interface given in Fig. 7. The MPS notifies the patient when the new SMS message is received. It filters the received SMS messages in two ways:

- The SMS messages received from the hospital server are filtered in the list box in the interface given in Fig. 7a. These messages have a specific format which starts with the symbol RPHMS\# and the SIM number inserted into the GPRS modem attached to the hospital server.

- The SMS messages that are received from other sources such as friends are not filtered within the healthcare messages as shown in Fig. $7 \mathrm{~b}$.

Although the mobile phone notifies the patient of receiving a new SMS message regardless of its source, there is a need to enable the patient to distinguish between the messages that come from the doctor and other messages.

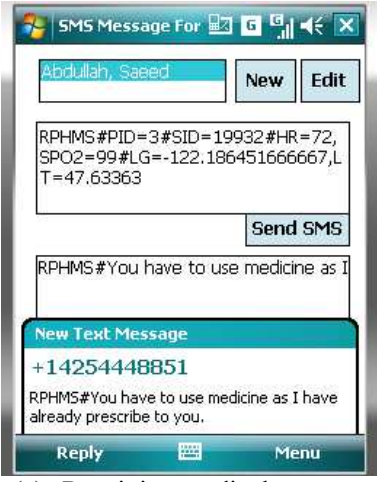

(a) Receiving medical message

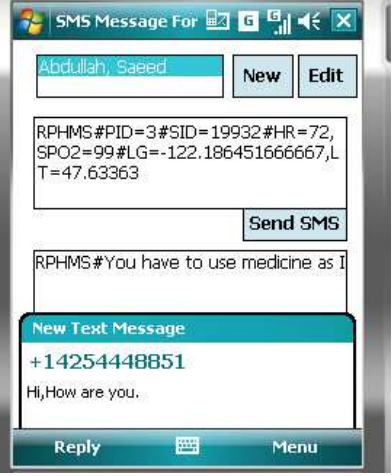

(b) Receiving other message
Fig. 7 Receiving the patient's feedback

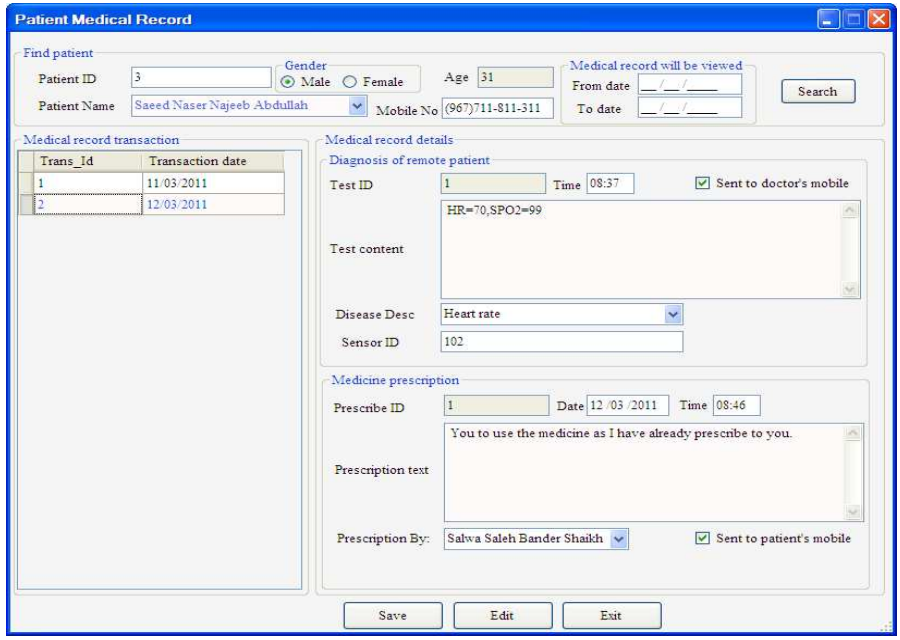

Fig. 8 Exploring current patient's location (CHS interface)

The Fig. 8 shows the patient's medical record, which is a document that includes all nursing and medical information that covering all aspects related to the patient's status. For viewing the patient's medical history, the doctor can find the specific patient by using the drop-down list in the interface given in Fig. 8. If the user wants to navigate the patient's medical record for specific duration, this can be done by entering the required duration and then click the button called search to display the patient's medical record. The interface given in Fig. 8 explains that the patient's medical record consists of three main parts: 
- The transaction of the medical record.

- Medical diagnosis of the patient. This section displays the patient's diagnostic data.

- The medical prescription of the patient.

This section displays the data of treatment and the necessary instructions to the patient described by the physician according to the patient's diagnostic data and the date and time of receipt from doctor's mobile phone and is this prescription sent to the patient or not.

Fig. 9 shows the interface of mapping of the current patient's location. This figure indicates that there are two parameters used to identify the current patient's location: latitude and longitude. The current values of the location's parameters are directly stored in the database of the CHS after receiving the current location data by the remote hospital server from the patient's mobile phone. Therefore, the user can navigate the current patient's location by looking for the required patient using the search criteria such as the patient's ID or patient's name in the drop-down list. After entering the search criteria, the system responds in displaying the current patient's location, then the user clicks the button called (map patient's location) to browse the current patient's location.

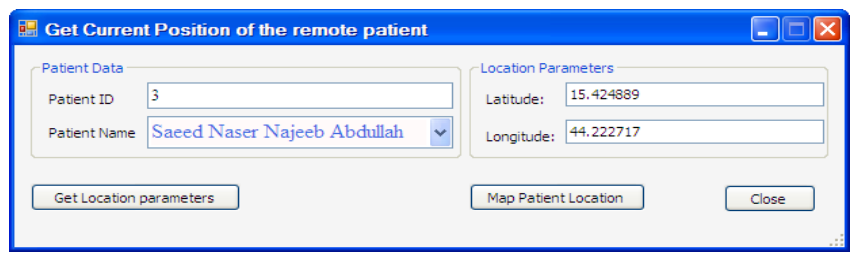

Fig. 9 Exploring Current Patient's Location (CHS Interface)

\section{Discussion}

The purpose of the evaluation is to present evidence that the RPHMS consistently provides the results, which meet the predetermined user requirements. To evaluate the RPHMS, several parameters need to be considered, such as user-friendly, system performance, and other innovative aspects. Firstly, the GUI of the MPS and CHS are reliable and user friendly. The users do not need to have more technical knowledge for using both MPS and CHS. The communication and application architecture of the RPHMS is appropriately flexible. The implementation of RPHMS is also perfectly soft. The MPS based mobile phone implementation does not need the patient interaction because of the following steps:

- The hospital system administrator in the hospital is responsible for installing the MPS in the patient's mobile phone and preparing the required communication settings to connect to a remote hospital server. This step will be made once after the patient's subscription.

- The sending and receiving operations are automatically done by the MPS.

- The MPS has an auto startup feature. This feature enables the MPS to start up when starting up the mobile device automatically.

For the CHS based hospital server implementation, the doctor interaction is optional because the doctor has the remote access privilege into the CHS through his/her mobile device. This permission enables the doctor to receive the patient's medical data and to make feedback anywhere and anytime. Secondly, several parameters are used to measure the system performance, such as the data storing, data receiving, data sending, and data retrieving process. The evaluation results for these parameters were referred that the CHS based hospital server implementation provides high performance due to its characteristics:

- It implemented oracle database $12 \mathrm{C}$, which provides high performance in responding to the system's SQL statements.

- The connection between GUI of the CHS and the database is implemented using the Oracle Data Provider (ODB.NET) which also provides highperformance data access

- The CHS is implemented and coded using the .NET threading technology, which also plays an essential role in increasing performance.

Furthermore, three innovative aspects distinguish the RPHMS as follows:

- The PSS is implemented as a subsystem of the CHS, which processes and manages all data and information related to the patient's subscription operation. It is considered as a valuable module from the healthcare provider point of view.

- The feedback must be sent from the doctor to the hospital server responsible for forwarding the patient's prescription to the patient's mobile phone.

It seems a long operation but it is better due to the feedback which must be authenticated and authorized by the hospital server. Then the feedback is stored in the patient's medical record before transferring it to the patient's mobile phone.

\section{CONCLUSION}

The RPHMS has been developed to facilitate the followup of chronic patients' status in remote areas that lack complete medical coverage. It is also designed to provide accurate and timely medical and location data to the hospitals and healthcare centers to assist with illness management. The RPHMS requirements were collected, studied, and analyzed. Then, the RPHMS design model was proposed and designed. In addition, the domain model, the Use-Case model, and the Use-Case specification model for the RPHMS were discussed. The system was designed in terms of UML and the activity diagram, sequence diagrams and class diagram were proposed and discussed. The whitebox and black-box were applied to test the functionality of the RPHMS, and show the system was operating correctly. The evaluation was done by measuring the following parameters: user-friendly, system performance, and other innovative aspects. Firstly, the system evaluation showed that both MPS and CHS were user friendly and reliable. Secondly, based on the system performance parameters, the system evaluation showed that both MPS and CHS provide good performance.

For further research, the researchers suggest further improvements in this research for remotely monitoring many chronic illnesses by utilizing new types of medical sensors. The PTS is used to track the location of the patients based on the GPS receiver. However, it receives the locations' signals from GPS satellites in an open environment. If the patient is 
located at home, then the current location's data cannot be measured. Accordingly, the researcher suggests improving the PTS for identifying patients' location in a wide range of areas, such as an indoors and outdoors tracking system based on mobile technology.

Moreover, the literature is still limited in the field of developing the PSS and the SMS management system as two subsystems of the remote healthcare monitoring systems. This was a limitation faced by the researcher during this study's execution. In the future, it is necessary to conduct more research in this field. Based on this research results, the researcher recommends the hospitals and healthcare centers in Yemen to implement the RPHMS. It was developed not only to improve the management of chronic illnesses but also to provide healthcare providers with financial value. Besides, the implementation of the RPHMS is very easy and not expensive because it uses cheap and commonly available technology. Furthermore, the researcher recommends the Ministry of Health in Yemen encourage and fund the implementation of the RPHMS in Yemeni hospitals.

\section{REFERENCES}

[1] N. K. Munjal and S. Ratan Singh, "Remote Health Monitoring System for Rural Areas," International Journal of Technical Research \& Science, vol. 5, no. 6, pp. 1-7, Jun. 2020.

[2] Majumder, S., Mondal, T., Deen, M.J. (2017). "Wearable Sensors for Remote Health Monitoring. Sensors", 17(1):130. Published 2017 Jan 12. doi: $10.3390 / \mathrm{s} 17010130$.

[3] Guo, M. (2020). "Application of Remote Sensing Technology in Macro-Ecological Environment Monitoring. Remote Sensing", 9(1), 27.

[4] Salehi, S., Olyaeemanesh, A., Mobinizadeh, M. et al. (2020). "Assessment of remote patient monitoring (RPM) systems for patients with type 2 diabetes: a systematic review and meta-analysis." Journal of Diabetes \& Metabolic Disorders.

[5] Kalid, N., Zaidan, A.A., Zaidan, B.B. et al. (2018). "Based Real Time Remote Health Monitoring Systems: A Review on Patients Prioritization and Related Big Data Using Body Sensors information and Communication Technology". J Med Syst 42, 30. https://doi.org/10.1007/s10916-017-0883-4.

[6] TECHALONE (2009). GPRS, retrieved from http://www.techalone.com/wp-content/uploads/2009/04/gprs.doc.

[7] Daely, P., Rizal, A. \& Hadiyoso, S. (2018). "Design of Wireless Vital Signs Monitoring System for Indoor Monitoring Activity". 7. 77-81. 10.14419/ijet. v7i4.44.26866.

[8] USTH (2018) Dataset: chronic diseases statistics.

[9] Andrews, L. J. B., et al. (2019). "Mobile android-based remote patient monitoring system through wearable sensors." Journal of Discrete Mathematical Sciences and Cryptography 22(4): 557-568.

[10] Kandukuri, K., Jency, A.A., Anita, R., Pavithra, K.S., Seema, V. (2015)."Android Based Women Safety Application with Blood Pressure, Heart Beat Monitoring and Location Tracking, Emergency
Support System", IJSRD - International Journal for Scientific Research \& Development, Vol. 3, Issue 01.

[11] Evans, J., Papadopoulos, A., Silvers, C. T., Charness, N., Boot, W. R., Schlachta-Fairchild, L., et al. (2016). "Remote Health Monitoring for Older Adults and Those with Heart Failure: Adherence and System Usability". Telemedicine journal and e-health: the official journal of the American Telemedicine Association, 22(6), 480-488. https://doi.org/10.1089/tmj.2015.0140.

[12] Izadi, V., Shahri, P.K. \& Ahani, H. (2020). "A compressed-sensingbased compressor for ECG. Biomed. Eng. Lett. 10, 299-307. https://doi.org/10.1007/s13534-020-00148-7.

[13] Mukherjee, R., Ghorai, S.K., Gupta, B. et al. (2020). "Development of a Wearable Remote Cardiac Health Monitoring with Alerting System." Instrum Exp Tech 63, 273-283.

[14] Baheti, P. K., Garudadri, H., \& Majumdar, S. (2010). "Low Complexity Sensors for Body Area Networks", PETRA'10, June 23 25, 2010, Samos, Greece.

[15] Nguyen, H. T. and T. V. Nguyen (2018). "Energy-Efficient and Low Complexity Channel Coding for Wireless Body Area Networks," 2018 5th NAFOSTED Conference on Information and Computer Science (NICS), Ho Chi Minh City, 2018, pp. 265-269, doi: 10.1109/NICS.2018.8606883.

[16] Djawad,Y.A., Suhaeb, S., Ridwansyah,Jaya, H., Fathahillah, Saharuddin. (2020). "Development of an intelligent mobile health monitoring system for the health surveillance system in Indonesia.".

[17] Zhang, Y., et al. (2015). "Remote Mobile Health Monitoring System Based on Smart Phone and Browser/Server Structure." Journal of Healthcare Engineering 6: 590401.

[18] Veyilazhagan, R. \& Dinesh, E. (2017): Real Time Health Care Patient Monitoring System Using GSM/GPS Technology, International Journal of Advanced Science and Engineering Research, Volume: 2, Issue: 1, April 2017.

[19] Murphy, J., et al. (2020)."Tracking physical activity using smart phone apps: assessing the ability of a current app and systematically collecting patient recommendations for future development". BMC Med Inform Decis Mak 20, 17 (2020). https://doi.org/10.1186/s12911-020-1025-3.

[20] Noimanee, S. \& Wattanasirichaigoon, S. (2008). "Implementation of Vital Signs Monitoring System Using Wireless Networks", International Journal of Applied Biomedical Engineering Vol.1, No.1.

[21] Valsalan, P., Baomar, T.A.B., Baabood, A.H.O. (2020). "IoT based health monitoring system." 7(4): 739-743.

[22] Kadhim, K.T., Alsahlany, A.M., Wadi, S.M. et al. (2020)."An Overview of Patient's Health Status Monitoring System Based on Internet of Things (IoT)." Wireless Personal Communications 114, 2235-2262.

[23] Cafazzo, J. A., Dunai, A, Feig, D. S., Hamill, M., Irvine, M. J., Logan, A.G., McIsaac, W.J., Tisler, A., Saunders, A., Rizo, C. A., \& Trudel, M., (2007). "Mobile Phone-Based Remote Patient Monitoring System for Management of Hypertension in Diabetic Patients. Mount Sinai Hospital", University of Toronto, 600 University Avenue, Suite 435, Toronto, Ontario M5G 1X5, Canada.

[24] Fareen, N. (2009)."Use of mobile messaging in health care". Allama Iqbal Open University, H-8 $\backslash 4$ Islamabad Pakistan.

[25] Oracle (2008). "Technical Comparison of Oracle Database $11 \mathrm{~g}$ and SQL Server 2008: Focus on Manageability". Oracle White Paper. Retrieved from https://www.oracle.com/technetwork/database/manageability/ss2008-vs-oracle-11g-tech-comparis-129127.pdf 